\title{
PCSK1 Variants and Obesity: Relationship in Different Population
}

\author{
Xiangtong Sha ${ }^{1 \mathrm{a}}$, Yueqiang Wang ${ }^{1 \mathrm{~b}}$ \\ ${ }^{a}$ Xiangtong Sha. Xiangya Medical College of Central South University, Changsha, Hunan province, China, 410000 \\ Email:8303181323@csu.edu.cn \\ ${ }^{b}$ Yueqiang Wang. University of York, Heslington, York, YO10 5DD, UK \\ Email:yw3372@york.ac.uk
}

\begin{abstract}
With the development of economy, obesity is now a common chronic disease which is becoming increasingly common all over the world. Up to $39 \%$ of adults worldwide are overweight. While 20 percent of children and adolescents, globally, are obese in 2020. Obesity can be affected by genetic factors, and PCSK1 was confirmed to be related to single gene obesity. PCSK1 encodes PC1/3 protein, and it is expressed not only in the peripheral nervous system and central nervous system but also in the endocrine and neuroendocrine organs. It regulates the production of several types of anorexia hormones and appetite-stimulating hormones, therefore, regulating food intake and body weight. It also plays a role in the endocrine system in regulating appetite, glucose homeostasis and nutrient assimilation. Thus, to figure out the effect of PCSK1 mutation on different humans' population and relationship between PCSK1 variants and obesity, this paper used the method of gathering studies of other scholars and analyzed the structure and function of PCSK1, as well as obesity degree of PCSK1 variation in different populations. Finally, it is concluded that common variants of PCSK1, such as rs6232 and rs6234/6235, were shown to be significantly associated with obesity risk, and these risks are strongly modulated by age and ethnicity. The results showed that PCSK1 had a greater effect on obesity in the young population, and rs6232 was associated with obesity in the white European population while rs6235 and rare mutations were associated with obesity in the Asian African population.
\end{abstract}

Keywords : Obesity, PCSK1 variant, Disease, Population

\section{INTRODUCTION}

Obesity is defined as an excess of fat body mass that can be harmful to one's health. Obesity is characterized by an energy imbalance induced by too much food and insufficient physical activity. According to the World Health Organization (WHO), obesity prevalence has risen worldwide since 1975. In 2016, approximately 1.9 billion adults were overweight, with 39 percent overweight and 13 percent obese. The body mass index (BMI), which is calculated by dividing weight by height $(\mathrm{kg} / \mathrm{m} 2)$, is used to categorize persons as obese (BMI greater than $30 \mathrm{~kg} / \mathrm{m} 2$ ). Furthermore, genetics has a role in the development of obesity, accounting for roughly $40 \%$ of the disease's heritability. Many researchers have studied obesity genes, but the issue is very complex and still needs more explorations and clearer summaries. Obesity was linked to mutations in these genes, such as PCSK1. In this paper, a collection of studies focusing on PCSK1 variations in various populations was compiled.
In this review, some aspects will be addressed, such as PCSK1 variants in children and adults. In addition, the functions of the principal gene PCSK1 that have been associated with obesity and PCSK1 variants associated with different ethnic groups are described. The significance of this paper is to provide reference for future studies in the academic field and help the general public better understand the relationship between genes and obesity.

\section{BASIC THEORY OF PCSK1}

\subsection{The Structure of Pcsk1}

PCSK 1 encodes the $\mathrm{PC} 1 / 3$ protein and is found on human chromosome 5q15-21. PC1/3 mRNA was transcribed from pcsk1, and subsequently ribosomes on the rough endoplasmic reticulum translated it into a 753residue protein (ER). The 753-residue protein is the inactive precursor signal peptide which called 
preproPC1/3. ProPC1/3 has four domains: a prodomain, a catalytic domain, a carboxy terminal domain, and a $\mathrm{P}$ domain that is exclusive to this superfamily. In many species and analogues, the catalytic domain is substantially conserved. The propeptide domain is responsible for the protein's proper folding and ER export. The $\mathrm{P}$ domain is required for the control of PCs' calcium and $\mathrm{pH}$ sensitivity, whereas the carboxy terminal domain aids in the sorting of $\mathrm{PC} 1 / 3$ into secretory granules[1].

\subsection{The Function of Pcsk1}

The expression of $\mathrm{PC} 1 / 3$ is limited to the peripheral and central nervous systems, endocrine and neuroendocrine organs. It is involved in the synthesis of several hormones and regulates appetite and energy metabolism.

\subsubsection{Pcsk1 Activates Hypothalamic neuropeptides}

In the brain, some areas of the hypothalamus express a lot of $\mathrm{PC} 1 / 3$, such as the arcuate nucleus, which is considered to have an important influence in regulating appetite and energy intake. The expression of hypothalamic $\mathrm{PC} 1 / 3$ exists in two neuronal populations: one is the POMC-expressing neurons, the other is the NPY and AgRP-expressing neurons, which are both leptin-sensitive. PC processes many hormones which participate in appetite regulation, such as a -MSH, $\mathrm{ACTH}$ and $\beta$-endorphin, they are all produced from POMC-expressing neurons. $\mathrm{PC} 1 / 3$ firstly mediates the production of adrenocorticotropic hormone (ACTH), then ACTH is specifically disintegrated and then finally modified to produce a-MSH. a-MSH is an effective anorexia hormone derived from pomc, which can reduce food intake and increase energy expenditure, and regulate glucose metabolism by activating melanocortin 4 receptor (MC4R) located in the paraventricular nucleus of the hypothalamus and other aim regions of the central nervous system[1].

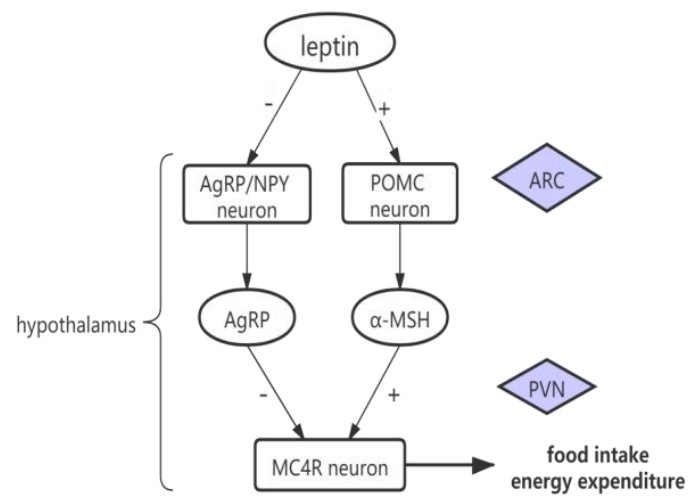

Figure1. Mechanism of leptin regulating appetite in hypothalamus
In the hypothalamus, leptin binds to receptors on AgRP neurons and POMC neurons, and inhibits AgRP neurons and stimulates POMC neurons. In this process, $\mathrm{PC} 1 / 3$ participates in the synthesis of a $-\mathrm{MSH}$ by POMC neurons and the processing of AgRP. The generated a-MSH and AgRP compete to bind to MC4R neurons in PVN. a -MSH stimulates MC4R neurons and increases appetite, while AgRP inhibits neurons and decreases appetite.

\subsubsection{PCSK1 in the Peripheral Endocrine System}

$\mathrm{PC} 1 / 3$ levels are also elevated in the peripheral organs such as pituitary, pancreas, stomach, and colon, as well as the thyroid glands and paranephros. $\mathrm{PC} 1 / 3$ is only expressed in the $\alpha$ - and $\beta$-cells of the islets, ghrelinexpressing gastric cells and different intestinal endocrine cells. Appetite, glucose metabolism and nutritional assimilation are all influenced by these specialised cells. $\mathrm{PC}$ and $\mathrm{PC} 2$ collaborate in the periphery to activate proinsulin and create mature insulin. Proglucagon is selectively converted to glucagon in pancreatic-cells that express PC2. In intestinal L cells missing PC2, PC1/3 is involved in glucagon processing to synthesize GLP-1 and GLP-2, which are known as glucagon-like peptides, but not glucagon itself[1].

\subsubsection{Other Appetite Peptides Regulated by PCSK1}

In addition, $\mathrm{PC} 1 / 3$ also participated in the synthesis of appetite suppressant CART expressed in POMC neurons. AgRP and NPY are orexigenic peptides that can stimulate appetite and reduce energy consumption, thus antagonizing the effect of POMC, and the synthesis of POMC also depends on PC1/3[1].

\section{THE EFFECT OF PCSK1 VARIANTS ON DIFFERENT POPULATION}

\subsection{Common PCSK1 Varieties}

In a number of human populations, PCSK1 is the third most prominent genetic factor to the risk of obesity. It contains many single nucleotide polymorphisms, the most common variants being rs6232 and rs6234/6235. The presence of an rs6232 allele was linked to lower oral glucose tolerance, lower insulin sensitivity, and higher proinsulin levels, whereas the rs6234/6235 variant was linked to lower fasting glucose and higher insulin levels [1]. The risk of both ordinary and extreme obesity was significantly associated with rs6232 and rs6234/6235, with age and race strongly regulating gene expression. 


\subsection{The Effect of Pcsk1 Variants on People of Different Ages}

PCSK1 variant was demonstrated to have an agerelated effect on obesity. According to studies, PCSK1 has a greater impact on obesity in children than it does in adults. Pieter Stijnen et al. found that the PCSK1 SNPs rs6232 and rs6234 - rs6235 play a minor but substantial role in the genetic susceptibility to obesity in an agedependent manner. They conducted a thorough review and meta-analysis of the current literature in Human Genome Epidemiology (HuGE). For the first time, this meta-analysis pooled data from 38 research cohorts from 19 studies, totaling over 200,000 people, and discovered that the link of PCSK1rs6232 with obesity is higher in juvenile cohorts than adult cohorts, as well as a similar effect of rs6234-6235[2].

According to Helene Choquet et al's research, the link between obesity and rs6232 may be age-related in European-Americans (hereinafter referred to as EA). They used data from two cohorts to investigate the role of common PCSK1 mutations in obesity in the American population: the Coronary Artery Risk Development in Young Adults (hereinafter referred to as CARDIA) group and the Multi-Ethnic Study of Atherosclerosis (hereinafter referred to as MESA). In EA cohort from CARDIA, researchers investigated the age-related influence of rs6232 on obesity and discovered that rs6232 was modestly linked with BMI in the younger age group but not in the older age group. They also got the same result by integrating samples of EA from both cohorts [3].

The correlation between PCSK1 and obesity is more significant in the younger population, and the evidence of influencing factors leading to this phenomenon is still unclear. Studies indicated that obesity-related genes are mainly expressed in the central system and are related to the increase of leptin level, suggesting that the mechanism of gene influence on childhood obesity may be related to leptin resistance in the central system, which also reflects the important role of central regulation on childhood obesity[4]. In addition, it may be related to feeding behavior. PCSK1 is known to regulate appetite, and the younger the population is, the more susceptible it is to the influence of appetite, thus changing feeding behavior.

\subsection{The Effect of Pcsk1 Variants on People of Different Ethnic Groups}

The impact of PCSK1 variation on obesity was likewise racially distinct. Helene Choquet et al. studied the influence of these two alleles on BMI variation and obesity risk in 1,320 EA of CARDIA and discovered that those with the minor rs6232 at-risk allele had a higher BMI. Only the rs6232 mutation was linked to an increased risk of obesity. Researchers next looked at the impact of rs6235 on 1,128 African-Americans (hereinafter referred to as AA) from CARDIA and discovered that those who had the lower rs6235 at-risk allele had a higher BMI. These findings suggest that the PCSK1 polymorphisms rs6232 and rs6235 play a moderate role in obesity in the CARDIA study [3].

There have also been a number of other epidemiological studies that have shown a link between PCSK1 polymorphisms and obesity. Kilpelainen TO et al. investigated young subjects (aged under 59 years) from Norfolk, UK, and discovered that rs6232 was associated modestly with BMI and obesity[5]. In 3,925 Danish participants, Sandholt $\mathrm{CH}$ et al. found a link between rs6232 and an increased risk of overweight, as well as a relationship between rs6235 and an enhancive risk of obesity[6]. In 979 Greek participants, Rouskas K confirmed the first link of rs6234 with obesity[7]. In 32,287 Europeans from 15 cohorts, the GIANT collaboration (Genetic Investigation of ANthropometric Traits) discovered a small link between rs6232 and BMI[8]. In a study of 1,094 Chinese people, Yi-Cheng Chang et al. found numerous common variations in PCSK1 (rs155971) that were linked to obesity[9]. Wen $\mathrm{W}$ et al. conducted a meta-analysis and found no indication of a link between rs6234 or rs6235 and BMI in 83,048 East Asians[10]. However, they did find a link between rs261967, which is near PCSK1, and BMI in 83,048 East Asians.

These studies showed different results indicating that PCSK1 variation does have a certain relationship with obesity, but rs6232, rs6234, rs6235 and other PCSK1 variations have different effects on different races. It can be found that rs6232 is mostly associated with obesity in people of European descent and caucasians, rs6234-6235 is mostly associated with obesity in African-Americans and Asians, and Asian obesity has also been associated with some less common PCSK1 variants. Due to the complexity of race, the effect of PCSK1 variation on obesity in different races needs further study.

\section{CONCLUSION}

Through the investigation and analysis of the effects of PCSK1 variations on different populations by different researchers, this paper sorts out the contribution of PCSK1 mutations to obesity on different human populations and the relationship between PCSK1 variants and obesity-related phenotype. It is confirmed that the evidence for the relationship of the PCSK1 variants (such as rs6232, rs6234 and rs6235) with overweight, obesity and BMI is complicated. The extent of the effect is related to race and age. In the last twenty years, research on PCSK1 SNPs and their mutations in different patient models help a lot to our current understanding of mono and polygenic PCSK1-dependent endocrinopathies. However, the shortcoming of this article is that it cannot fully describe the impact of this gene on other groups of people. In comparison with 
research on single ethnic groups, this paper will contribute to future research on the effects of PCSK1 on multi-ethnic groups. It is expected that future research will focus on figuring out the specific mechanisms by which the PCSK1 variant affects different populations, in order to better target the treatment of obesity.

\section{ACKNOWLEDGMENTS}

We profited from the presence of our teachers Anny and Sherry while composing this essay. They gladly provided us with numerous helpful ideas. We would want to offer our heartfelt gratitude to them for their invaluable assistance, without which the paper would not have been completed. Special thanks to Xiangtong Sha, who has done a lot of material collection and data statistical analysis for the completion of the paper, and completed most of the paper writing. Special thanks to Yueqiang Wang for his overall review and contribution to the paper.

We owe a debt of gratitude to our parents, who provided us with a lot of encouragement and financial support while we were working on our thesis. They always share our joys and sorrows with us. We are deeply grateful to them and owe our success to them.

\section{REFERENCES}

[1] B. Ramos-Molina, M.G. Martin, I. Lindberg, PCSK1 Variants and Human Obesity. Progress in molecular biology and translational science, 2016, 140, 47-74.

[2] P. Stijnen, K. Tuand, T.V. Varga, P.W. Franks, B. Aertgeerts, J.W. Creemers, The association of common variants in PCSK1 with obesity: a HuGE review and meta-analysis. American journal of epidemiology, 2014, 180(11), 1051-1065.

[3] H. Choquet, J. Kasberger, A. Hamidovic, E. Jorgenson. Contribution of common PCSK1 genetic variants to obesity in 8,359 subjects from multiethnic American population. 2013, PloS one, 8(2), e57857.

[4] J.L. Fu, Exploration of the relationship between obesity-related genes, adipokine profiles and metabolic phenotypes in children. (Master dissertation, Peking Union Medical College). 2017, https://kns.cnki.net/KCMS/detail/detail.aspx?dbna me $=$ CMFD201801\&filename $=1017225171 . \mathrm{nh}$

[5] T.O. Kilpeläinen, S.A. Bingham, K.T. Khaw, N.J. Wareham, R.J. Loos. Association of variants in the PCSK1 gene with obesity in the EPIC-Norfolk study. Human molecular genetics, 2009, 18(18), 3496-3501.

[6] C.H. Sandholt, T. Spars $\varnothing$, N. Grarup, A. Albrechtsen, K. Almind, L. Hansen, U. Toft, T. Jørgensen, T. Hansen, O. Pedersen, Combined analyses of 20 common obesity susceptibility variants. Diabetes, 2010, 59(7), 1667-1673.

[7] K. Rouskas, A. Kouvatsi, K. Paletas, D. Papazoglou, A. Tsapas, S. Lobbens, V. Vatin, E. Durand, Y. Labrune, J. Delplanque, D. Meyre, P. Froguel, Common variants in FTO, MC4R, TMEM18, PRL, AIF1, and PCSK1 show evidence of association with adult obesity in the Greek population. Obesity (Silver Spring, Md.), 2012, 20(2), 389-395.

[8] C.J. Willer, E.K. Speliotes, R.J. Loos, S. Li, C.M. Lindgren, I.M. Heid, S.I. Berndt, A.L. Elliott, A.U. Jackson, C. Lamina, G. Lettre, N. Lim, H.N.Lyon, Genetic Investigation of ANthropometric Traits Consortium. Six new loci associated with body mass index highlight a neuronal influence on body weight regulation. Nature genetics, 2009, 41(1), 25-34.

[9] Y.C. Chang, Y.F. Chiu, K.C. Shih, M.W. Lin, W.H. Sheu, T. Donlon, J.D. Curb, Y.S. Jou, T.J. Chang, H.Y. Li, L.M. Chuang, Common PCSK1 haplotypes are associated with obesity in the Chinese population. Obesity (Silver Spring, Md.), 2010, 18(7), 1404-1409.

[10] W. Wen, Y.S. Cho, W. Zheng, R. Dorajoo, N. Kato, L. Qi, C.H. Chen, R.J. Delahanty, Y. Okada, Y. Tabara, D. Gu, D. Zhu, X.O. Shu, Meta-analysis identifies common variants associated with body mass index in east Asians. Nature genetics, 2012, 44(3), 307-311. 